\title{
Correction
}

\section{Correction: Shi et al., miR-9 and miR-140-5p Target FoxP2 and Are Regulated as a Function of the Social Context of Singing Behavior in Zebra Finches}

In the article "miR-9 and miR-140-5p Target FoxP2 and Are Regulated as a Function of the Social Context of Singing Behavior in Zebra Finches" by Zhimin Shi, Guanzheng Luo, Lijuan Fu, Zhide Fang, XiuJie Wang, and XiaoChing Li, which appeared on pages 1651016521 of the October 16, 2013 issue, the authors regret that not all the funding sources were cited in the accepted version of the Acknowledgments. The corrected Acknowledgments are as follows: This work was supported by NIH Grant R21 MH081254, NSF Grant IOS-1258015, and a Young Investigator Award from Brain and Behavior Research Foundation to X.C.L.

DOI:10.1523/JNEUROSCI.1701-14.2014 